\title{
The stability and eigen oscillations of sunspots
}

\author{
A.A. Soloviev and E.A. Kirichek \\ Central (Pulkovo) astronomical observatory, St-Petersburg \\ email: solov@gao.spb.ru
}

\begin{abstract}
One of the observational facts about sunspots is that magnetic field strength of sunspots varies in narrow interval $\approx$ from 1 to $4-5 \mathrm{kG}$. Why sunspot equilibrium is stable only under these values? To answer this question, we propose the energetic model of sunspot, which involves the gravitational energy of the system. Formation of a sunspot is followed by redistribution of gas density along the vertical: cooled plasma flows down, inside the "magnetic funnel", from above the sunspot to the "low boundary of magnetic flux tube", i.e. to the region where the magnetic field of sunspot decreases abruptly with depth. Theoretical model gives the interval of stable sunspot equilibria exactly between $0.8-1.0$ and $4-6 \mathrm{kG}$, which suites the observations. The "depth of sunspot" turns to be about $2-4 \mathrm{Mm}$ - this fact now is approved by helioseismology. Also, the long-term $(\mathrm{T}>20 \mathrm{~min}$ ) eigen oscillations of sunspot, as a whole, are studied. These oscillations were measured by different methods, being observed as quasi periodical vertical displacements of the body of sunspot followed by variations of its radius and magnetic field strength.
\end{abstract}

Keywords. sunspots, magnetic fields, MHD

In the present, we have a number of observations of long-term oscillations of sunspots as a whole (Gelfreikh et al. (2004), Gelfreikh et al. (2006)), which we call eigen oscillations of a sunspot. Traditionally, a simple model is used to describe sunspot oscillations: vertical magnetic flux tube with only field component $B=B_{z}$. This model can be applied when analyzing the observed short-term (3-5 min) oscillations in the sunspots (see Bogdan (2000)). This very simple model, although, does not allow to explain i) stability of the system as a whole, and ii) long-term ( $\mathrm{T}>20 \mathrm{~min}$ ) eigen oscillations of sunspots. To describe these phenomena one should take into account not only the magnetic field strength inside the sunspot $B$, but also another basic parameters of sunspot, like: vertical scale of magnetic field over the sunspot $\lambda_{m}$; Wilson's depression $\xi(B)$; radius of sunspot umbra $a$; external magnetic field $\left.B_{0}(\cong 0.25 k G)\right)$ and, finally, the finite "depth of sunspot" $l$. (The last is the depth of the layer, where the magnetic flux tube of sunspot expands rapidly downwards. This depth turns to be small enough, being about 3-4 Mm (Fig. 1). (Much before, basing on theoretical analysis of torsional oscillations observed in bipolar group of sunspots, it was shown (Soloviev (1984)) that the sunspot is a very "shallow" structure. Later this result was confirmed by other authors, and now it could be obtained "directly" by helioseismology methods (Zhao et al. (2001))).

Three basic properties of sunspot are: 1) cooling followed by radial constriction; 2) fan structure of magnetic field over sunspot umbra, that results in penumbra formation; 3 ) geometrical depression of visible layers, $\tau \cong 1$. These properties are physically bind into one process of vertical redistribution and lateral compression of sunspot gas, when it cools down. The dislocation of plasma from the funnel over the sunspot umbra to the depth $l$ decreases the gravitational energy of the system and stipulates stability of the system. Under lateral compression and immersion of the spot into the dense layers, the magnetic energy of the system increases. Therefore, the stability of sunspot is determined 

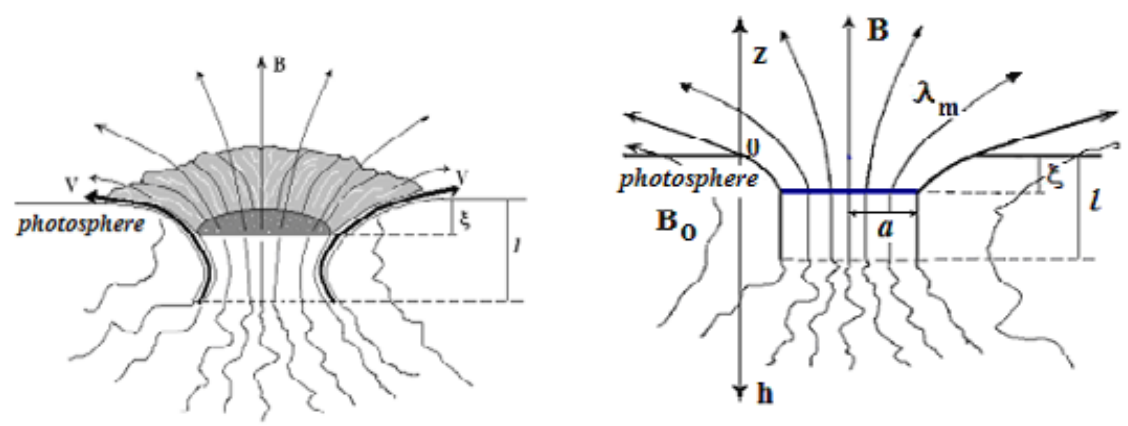

Figure 1. Real structure of sunspot magnetic field (left), and the theoretical model of "shallow sunspot" (right). Height h of cylindrical part of flux tube lies between the depths $\xi<h<l$.

by the balance between the increase/decrease of magnetic energy and decrease/growth of gravitational one during the process of immersion/ascent of the sunspot. It is evident that vertical displacements are followed by variations of magnetic field strength in the observable layers: when magnetic tube of sunspot arises and expands, the field $B$ decreases; when the tube sinks and compresses, $B$ grows. The analytic expression for dependence of Wilson's depression $\xi(B)$ on magnetic field is derived from the pressure balance on the upper edge of magnetic cylinder, $P_{e x}(\xi)=R \rho(\xi) T_{S, \text { ef }} / \mu+B^{2} / 8 \pi$, using the photosphere and convective zone models, as well as the empirical dependence of effective temperature on magnetic field strength, obtained by Kopp et al. (1992).

We use the energetic method to describe the equilibrium and stability of the system. The first variation of the energy, taken under condition of conservation of magnetic flux $\Phi=\pi a^{2} B$ and mass $M$ of sunspot, gives the equilibrium of sunspot: $\delta E=0$. The variations of basic parameters are connected by relations: $\delta B / B=-2^{\delta a} / a$ (if $\Phi=$ const), and $-2^{\delta a / a}=q(l) \delta l / l$ (if $\mathrm{M}=\pi a^{2} \int_{0}^{l} \rho_{0}\left(1+2.53 h+1.22 h^{2}+3.27 h^{3}\right) d h=$ const), where $q(l)$ is determined by density distribution in the upper part of the convective zone and varies from 1 to 4 . The second variation $\delta^{2} E \propto \delta l^{2}$, taken near the equilibrium state, gives the potential energy of the system, and correspondingly, the frequency of eigen vertical-radial oscillations of sunspot.

$$
\omega(B)=\frac{2.824 \cdot 10^{-6} B}{Z \sqrt{\rho_{0}}} \sqrt{\frac{Z\left(2+\frac{1}{q}-\frac{Z}{q} \frac{d q}{d l}\right)-3\left(1-\frac{k}{2}\right) B \frac{\partial \xi}{\partial B}-B^{2}\left(1-\frac{k}{2}\right) \frac{\partial^{2} \xi}{\partial B^{2}}}{l\left(1+1.265 l+0.407 l^{2}+0.8175 l^{3}\right)}}
$$

Here $Z \equiv \frac{l}{q}=\frac{\lambda_{m, 0} B_{0}^{2}}{2 B^{2}}+\left(1-\frac{k}{2}\right) B \frac{\partial \xi}{\partial B} ; k$ is a small constant $(\approx 0.1) ; \rho_{0}$ is plasma density in the photosphere; $\lambda_{m, 0}$ is the magnetic scale of external magnetic field $(\cong 0.7 \mathrm{Mm})$.

Theoretical dependence was calculated for two basic modes: for the oscillations with the $\mathrm{M}=$ const, and for mode with the fixed depth $\mathrm{l}$ (the effective mass is less then $\mathrm{M}$ ). These curves fit well the observational data obtained at Pulkovo observatory. The periods of the measured eigen long-term oscillations of sunspots vary from 40 to 200 minutes.

\section{References}

Gelfreikh G.B. et al. Proc. of IAU Symp. 223. St.-Petersburg. 2004. P. 525

Gelfreikh G.B., Nagovitsyn Yu.A., Nagovitsyna E.A. Publ. Astr. Soc. Japan, 2006. V.58. P. 29 Bogdan T.J. Sunspot oscillations: a review. Solar Phys. 2000. V. 192. P. 373

Soloviev A.A., Solnechnye dannye, 1984, No 1, P. 73

Zhao J., Kosovichev A.G, Duval T.L. Ap. J. 2001. V. 557. P. 384

Kopp G., Rabin D. Solar Phys. 1992. V. 141. P. 253 